Faculty of Science

Faculty Publications

This is a post-review version of the following article:

Method for Studying High Temperature Aqueous Electrochemical Systems: A Self Pressurized Autoclave

Thomas Holm, Per Kristian Dahlstrøm, Svein Sunde, David A. Harrington and Frode Seland

2016

The final published version of this article can be found at:

http://dx.doi.org/10.1149/07514.1055ecst

Citation for this paper:

Holm, T., Dahlstrøm, P.K., Sunde, S., Harrington, D.A. \& Seland, F. (2016). Method for Studying High Temperature Aqueous Electrochemical Systems: A SelfPressurized Autoclave. ECS Transactions, 75(14), 1055-1061. 
Accepted version of ECS Transactions 75 (2016) 1055-61. doi: 10.1149/07514.1055ecst

(C) The Electrochemical Society, Inc. 2016. All rights reserved. Except as provided under U.S. copyright law, this work may not be reproduced, resold, distributed, or modified without the express permission of The Electrochemical Society (ECS).

\title{
Method for Studying High Temperature Aqueous Electrochemical Systems: A Self-Pressurized Autoclave
}

\author{
T. Holm ${ }^{\mathrm{a}, \mathrm{b}}$, P.K. Dahlstrøm ${ }^{\mathrm{a}, \mathrm{b}}$, S. Sunde ${ }^{\mathrm{a}}$, D. A. Harrington ${ }^{\mathrm{b}}$, F. Seland ${ }^{\mathrm{a}}$ \\ ${ }^{a}$ Department of Materials Science and Engineering, Norwegian University of Science \\ and Technology (NTNU), NO-7491 Trondheim, Norway \\ ${ }^{\mathrm{b}}$ Department of Chemistry, University of Victoria, Victoria, British Columbia, V8W 3V6, \\ Canada
}

\begin{abstract}
A detailed description of a method using a self-pressurized autoclave to run aqueous electrochemical experiments at temperatures above the normal boiling point of water is given. Relevant challenges with studying electrochemical reactions at high current densities in such systems are identified, and potential methods for solving these challenges are provided. The method is demonstrated for a three-electrode setup in sulfuric acid electrolyte up to $140^{\circ} \mathrm{C}$.
\end{abstract}

\section{Introduction}

The ability to perform reproducible aqueous electrochemistry at temperatures above the normal boiling point of water is an important step to increase the efficiency of processes that are slow at and around room temperature. High temperature aqueous electrochemistry has been reviewed (1-4) and allows for a study of processes at fast reaction rates and allows for derivation of temperature dependent kinetic parameter. This type of electrochemistry has relevance for the study of corrosion (5), reference electrodes (6-8), $\mathrm{pH}$ measurements (9-10), transport properties (11-13), and nuclear materials (14), while the particular method used here builds on previous work where pressurized autoclaves have been used to study alcohol oxidation (15-17).

This work discusses in detail the problems arising when attempting to increase the temperature above the normal boiling point of water. In addition, a full setup consisting of a self-pressurized autoclave is described that has been used successfully to study methanol and glycerol oxidation and the surface processes on platinum electrodes in 0.5 M sulfuric acid solution. The experimental setup described in this work can easily be expanded to systems using other solutions and electrodes.

\section{Experimental}

The three-electrode self-pressurized autoclave system consisted of a $300 \mathrm{~mL}$ glass autoclave with a PTFE lid and metal tightening system (all Büchi Glas Uster AG) as shown in Fig. 1. The autoclave had four openings and used for three Pt electrodes and one manometer, and could be used for pressures up to 10 bars. The working electrode and counter electrode were glass-sealed Pt wire electrodes, where the counter electrode had a Pt mesh welded to the wire. The reference electrode was a reversible hydrogen electrode (RHE) consisting of a platinum mesh that is inside a tube of glass and sealed at the top. This configuration for the reference electrode allowed for easy production of $\mathrm{H}_{2}$, which 
can be collected in the tube to create the equilibrium between $\mathrm{H}^{+}$in the solution and the hydrogen gas. The reference electrode was kept in a glass compartment in a solution of $0.5 \mathrm{M} \mathrm{H}_{2} \mathrm{SO}_{4}$, separated from the main electrolyte using a ground glass joint.

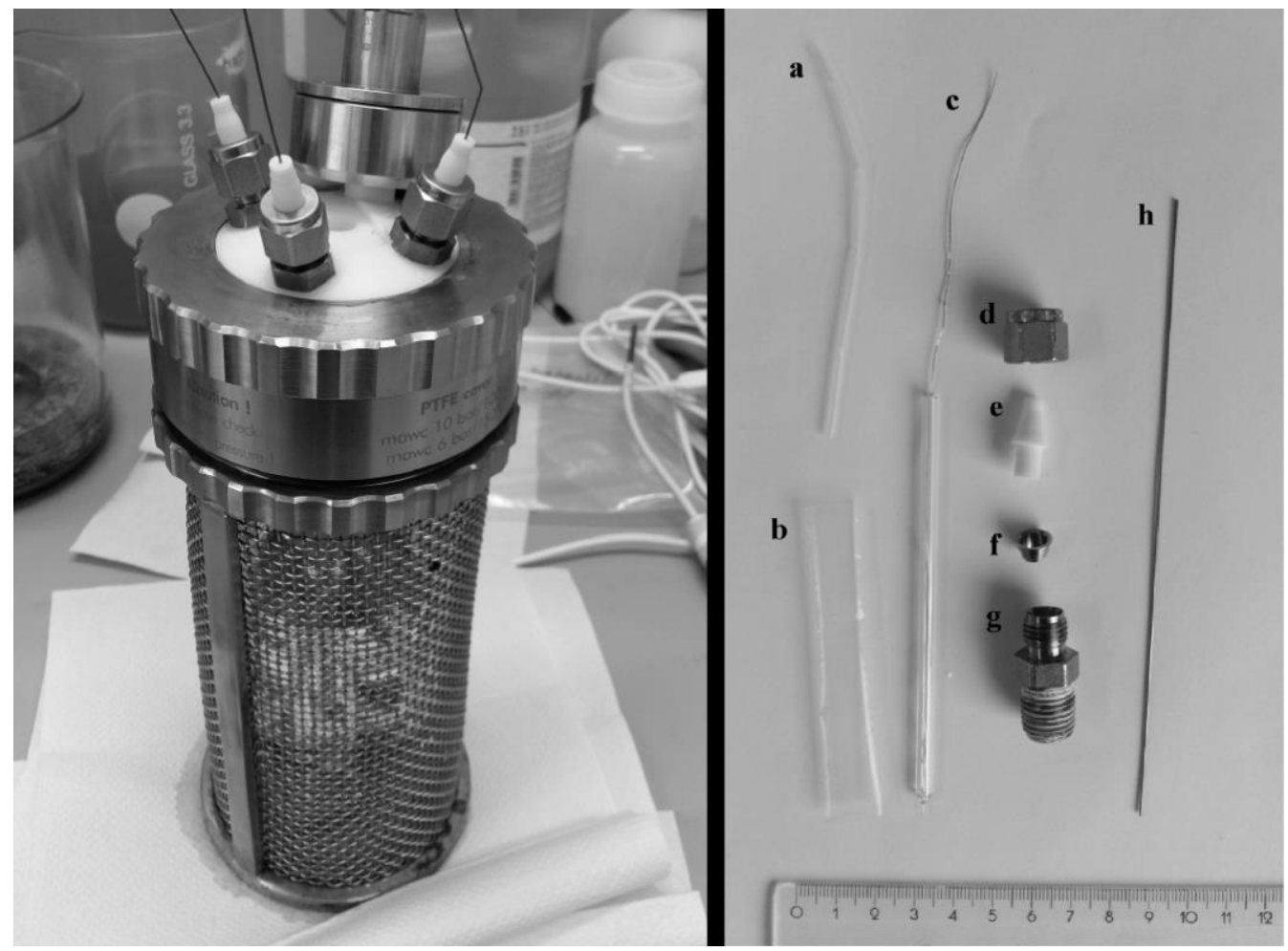

Figure 1. Left: The full autoclave setup with three electrodes and manometer. Right: An example of the parts making up a full electrode that can be sealed off. The parts are PTFE heat-shrinkable tubing a) $3.2 \mathrm{~mm}-1 \mathrm{~mm}$ and b) $9.5 \mathrm{~mm}-2.4 \mathrm{~mm}$, c) the electrode with platinum wire sealed in $4 \mathrm{~mm}$ outer diameter glass, d) top nut, e) PTFE wire seal piece, $\mathrm{f}$ ) sealing cone, g) bottom nut, and h) twist free piano wire for a proper seal.

The electrical connection to the outside of the autoclave was made possible by a twist free piano wire (KI\&S Engineering $54500-6 \mathrm{~mm}$ ). The sealing around this wire was done by a custom-made sealing piece in polytetrafluorethylene (PTFE). This sealing piece was fitted in a 1/4 inch connection (full set in Nickel-Copper Monel from Swagelok: M-4001-4). PTFE heat-shrinkable tubing was used to keep the electrode wires separate from the electrolyte. The whole arrangement for one electrode consisted of the various parts displayed in Fig. 1.

The system was heated by a hot plate (Torrey Pines Scientific HP61A or IKA CMAG HP10), and heating oil stable to temperatures up to $210^{\circ} \mathrm{C}$ was used (Dow Corning $210 \mathrm{H}$ Fluid). The system was calibrated before runs because the temperature could only be measured in the oil during experiments. This was done by heating the autoclave at intervals with the same amount of pure water (Millipore Milli-Q) as electrolyte in later experiments and recording the temperature in the electrolyte with a thermometer that was fitted to the connections used (VWR Traceable Dual-Channel Thermometer no 23609230). 


\section{Results and Discussion}

\section{Effect of Sealing Quality}

To pressurize an autoclave, it is obvious that a good seal is necessary to obtain a high pressure. Poor seal quality can be detrimental to the response over time, as evaporation of the active component can lead to a decline in the response. In particular, this is a problem when using an active component with a higher partial pressure than water at high temperatures. An example of this is methanol, which in pure form has a normal boiling point of $64.7^{\circ} \mathrm{C}$, and a partial pressure at $100^{\circ} \mathrm{C}$ of 3.4 bar. This leads to a selective loss of the electrochemically most active component of the electrolyte and gives a poor and irreproducible results. An example of loss of methanol is shown in Fig. 2 where the activity is reduced by about $90 \%$ over the duration of the experiment (223 $\mathrm{min}$ ).

Quantification of the actual loss is not straightforward, so experiments of similar quality as displayed in Fig. 2 were not utilized for any quantitative analysis. This specific problem was solved by improving the seals using the electrode setup in Fig. 1. The key improvement was to replace the easily dented Pt wired connection through the seal piece with a straight, strong and electrically conducting wire (Fig. 1h). This straight and strong wire, combined with the PTFE sealing piece, allowed for an excellent seal of the $1 / 4$ inch connection (Fig. 1d,g). This arrangement ensured efficient operation up to $150^{\circ} \mathrm{C}$ with a stable pressure without notable loss of electrolyte components.

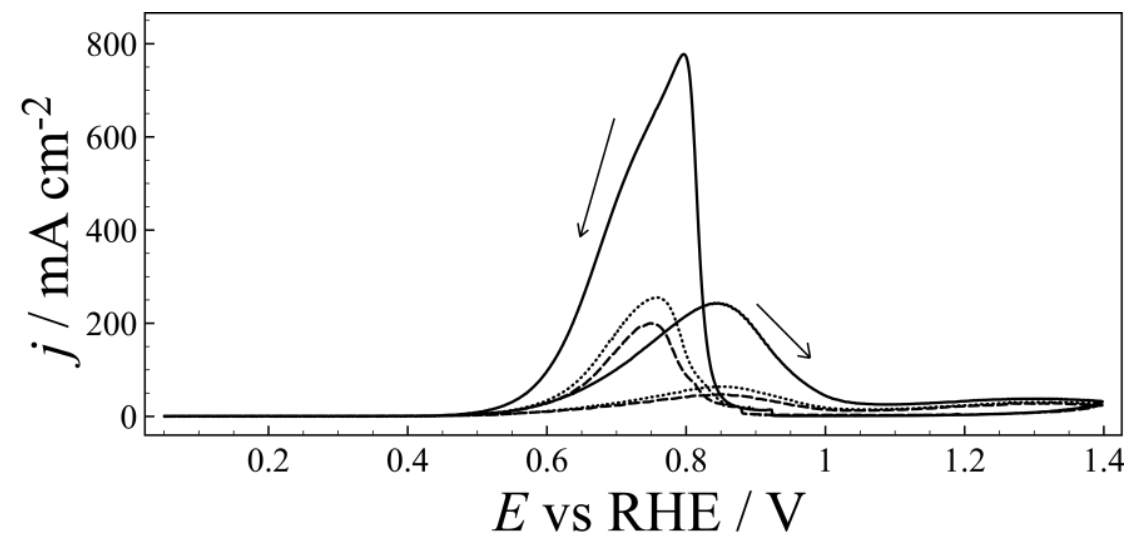

Figure 2. An example of the decline in current density over time due to evaporation of methanol from the electrolyte solution. All cyclic voltammograms are measured using the same setup at different times; $t=0 \mathrm{~min}$ (full line), $t=180 \mathrm{~min}$ (stippled line), and $t=223$ min (dashed line), the sweep rate was $200 \mathrm{mVs}^{-1}$ and the temperature was $140^{\circ} \mathrm{C}$.

\section{$\underline{\text { Ohmic Resistance Effects }}$}

Ohmic resistance is an important parameter in all electrochemical measurements and should normally be estimated and compensated for. This effect is related to the potential drop due the electrolyte resistance between the working electrode and the reference electrode. In our experiments, a linear potential current line was observed in high temperature experiments. This effect could not be compensated for by estimating the solution resistance, and was only observed very high total currents as would be expected for $I R$ drop effects. The effect is seen in Fig. 3, where (a) has a small electrode size and a 
reasonable result, while (b) has a large electrode $\left(3 \mathrm{~cm}^{2}\right)$ (18). The larger electrode leads to a linearized current response due to the total current between the working and the counter electrode will reach the limit of the electrolyte. Similar behavior was observed by Umeda et al. (19) and Bokach et al. (20) studying organic fuel oxidation in acidic electrolyte at Pt catalysts at elevated temperatures and high concentrations of organic fuel. It is likely that the high area of the catalyst electrodes leads to erroneous voltammetric responses at elevated temperatures. This is hinted at by Bokach et al. who also performed experiments with low concentration of methanol, resembling the familiar voltammogram expected for methanol at Pt electrodes.

Fig. 3 illustrates the solution to the problem of large ohmic resistances. This is simply to use a relatively small electrode. While a quantitative criteria for a good experimental setup was not determined, a qualitative indication that high temperature peaks should have a similar shape as room temperature peaks and that the presence of linear current vs potential plots are a good indicator that ohmic resistance or too large working electrode area may be causing a problem. Naturally, this is only a problem for very high currents. Therefore, it is dependent on the system studied and the magnitude of currents that are expected. Therefore, the general rule of thumb is to use as small electrode as possible provided the small currents can be measured without too much noise.

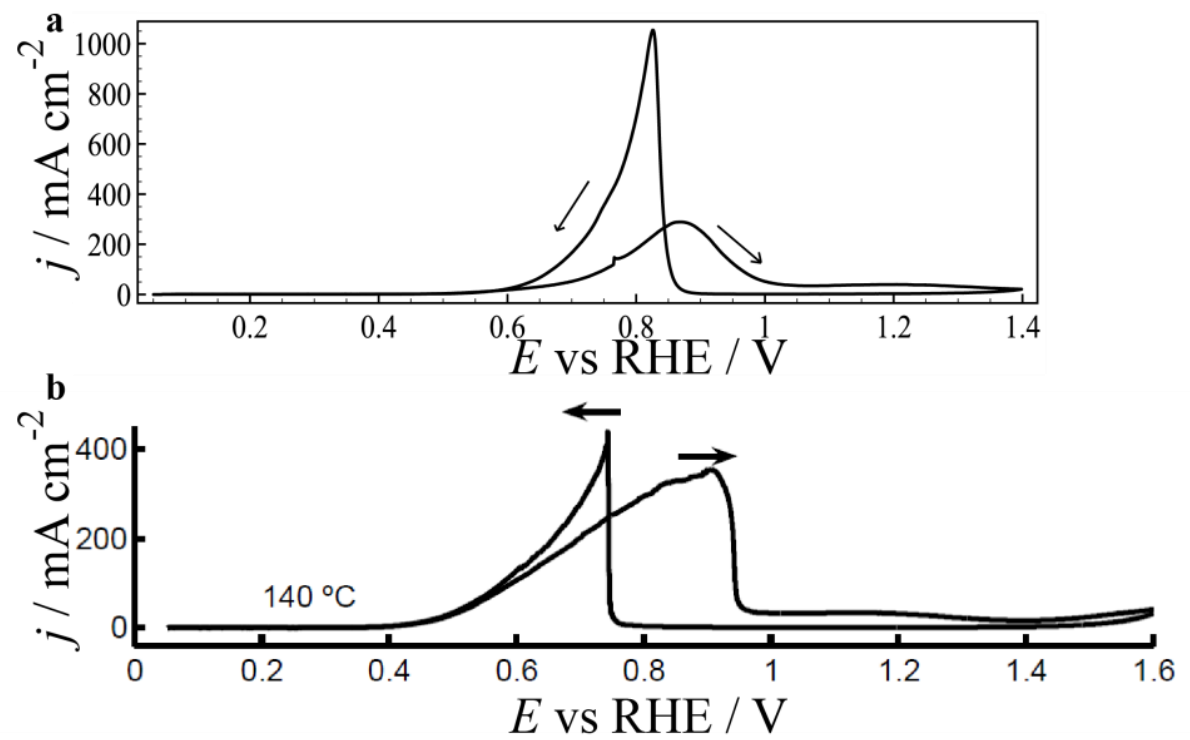

Figure 3. Example of a (a) small electrode $\left(0.005 \mathrm{~cm}^{2}\right)$ and a (b) large electrode $\left(3 \mathrm{~cm}^{2}\right)$ performing cyclic voltammetry at low sweep rates $\left(\mathrm{a}-20 \mathrm{mV} \mathrm{s}^{-1}\right.$ and $\left.\mathrm{b}-10 \mathrm{mV} \mathrm{s}{ }^{-1}\right)$ at $140^{\circ} \mathrm{C}$. The linear slope visible when using the large electrode is primarily caused by the high electrolyte IR drops. The data for the large electrode is adopted from a previous work with the autoclave (18). Note the different current scale.

\section{Stability of the Electrochemically Active Area}

A small electrode is an advantage in electrochemical experiments and a necessity for reactions with large current densities. However, temperature changes and preparation process can influence the electrode size in ways not intended. For a thin platinum wire electrode that is polished as a disc sealed in glass, the weak molecular interactions between platinum metal and glass as well as the fact that the thermal expansion of the 
platinum and the glass does not scale with each other, can be problematic. Still, obtaining a good seal between the Pt wire and the glass is essential in minimizing the electrode area and avoid seal failure exposing more metal to the electrolyte. Ultimately, repeated heating and cooling of the seal will lead to seal damage through formation and propagation of small cracks where electrolyte potentially can intrude and effectively change the active working electrode area. This effect was observed for a $10 \mu \mathrm{m}$ diameter commercially obtained microelectrode (BASi MF-2005) as shown in Fig. 4. After the electrode had been heated to $140^{\circ} \mathrm{C}$ (stippled line) and $160^{\circ} \mathrm{C}$ (full line), the electrode was much larger than the electrode as new (dash-dot line). This was determined from the area of the hydrogen adsorption peaks during cyclic voltammetry in $0.5 \mathrm{M} \mathrm{H}_{2} \mathrm{SO}_{4}$ at room temperature. Such an increase in electrochemically active area is detrimental in the study of temperature effects of electrochemical reactions.

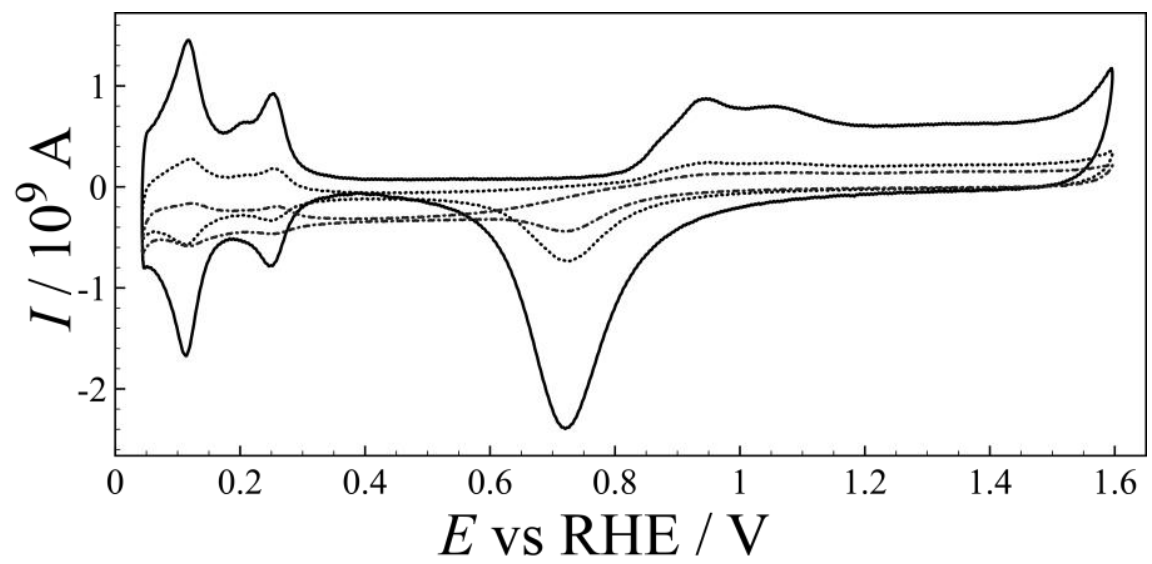

Figure 4. Example of electrode growth when using a $10 \mu \mathrm{m}$ microelectrode. Room temperature cyclic voltammetry at the first cycle (dash-dot line), room temperature cyclic voltammetry after the first heating cycle (stippled line) (max. temp $140^{\circ} \mathrm{C}$ ), and room temperature cyclic voltammetry after the second heating cycle (full line) are shown (max. temp $160^{\circ} \mathrm{C}$ ). Sweep rates are at $100 \mathrm{mV} \mathrm{s}^{-1}$.

In addition to a larger active electrode area resulting from electrolyte intrusion in small gaps between the electrode and the seal material, the possibility of accumulating product gas within the cracks is also plausible. Such an effect was consistently seen for electrodes with an increased working electrode area, in particular during the negativegoing sweep at the onset of platinum reduction as shown in Fig. 5. This effect led to a large increase in the current that quickly descended to trace a current perhaps resulting merely from the electrode area confined to the outside of the sealed area, i.e. the flat ground original area.

The problems associated with higher temperatures yielding an increased electrochemically active area, and with cracks giving artifacts in the cyclic voltammogram can be solved by adjusting the working electrode geometry. More specifically, the problem is reduced by using a wire electrode where the fraction of the area associated with the seal is minimized, i.e., by using an electrode with greatly increased length/diameter ratio. Therefore, the design of any high temperature experiment should incorporate all effects related to the electrode size meaning that the electrode size should be minimized while most of the electrochemically active area should be associated with the surface of the wire and not be greatly influenced by any cracking in the 
platinum-glass seal. A satisfying compromise was found in this work by employing a $0.05 \mathrm{~mm}$ diameter Pt wire (VWR 99.95\%) and allowing for 3-5 $\mathrm{mm}$ of the Pt wire to be kept outside the seal, allowing for a high length/diameter ratio and minimizing seal effects in the experiments with the highest current densities.

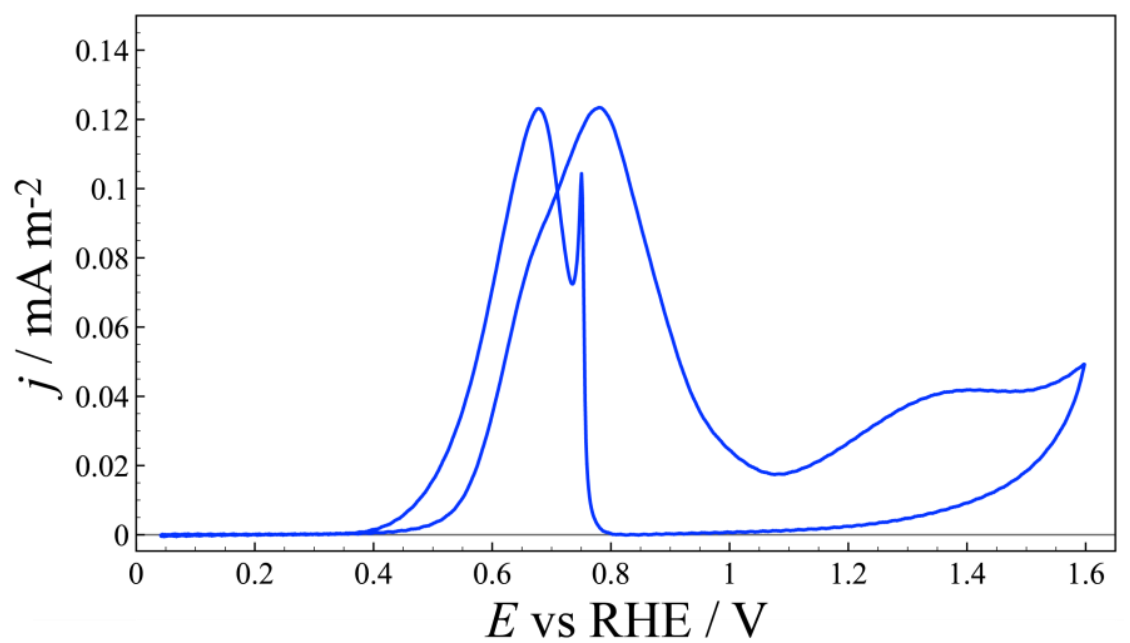

Figure 5. Example of downward sweep giving an extra peak due to poor seal between platinum and glass. Sweep rate is $1000 \mathrm{mV} \mathrm{s}^{-1}$ and temperature is $120^{\circ} \mathrm{C}$.

\section{$\underline{\text { Reference Electrode Stability }}$}

As the reference electrode is inside the autoclave, the potential is influenced by the temperature change. However, to keep the change predictable, the partial pressure of hydrogen must be kept constant at each temperature. If this criterion is not met, a drift in the reference electrode potential can be observed. Therefore, the autoclave heat gradients should be minimized, and this translates to a low heating rate. From experience in operating the system, a heating rate of less than $20 \mathrm{~K} \mathrm{hr}^{-1}$ is recommended.

\section{Conclusions}

A successful experimental apparatus and procedure was established for conducting high temperature aqueous electrochemical experiments. Specific challenges associated to ohmic resistance, working electrode area, and reference electrode stability are discussed. A compromise using a thin $\mathrm{Pt}$ wire with a high length/thickness ratio for high current measurements, and slow heating/cooling rate are recommended for optimum operation.

\section{Acknowledgments}

This research was partly conducted under the Engineered Nickel Catalysts for Electrochemical Clean Energy project administered from Queen's University and supported by Grant No. RGPNM 477963-2015 under the Natural Sciences and Engineering Council of Canada (NSERC) Discovery Frontiers Program. Financial support was also provided from the NSERC (discovery grant 37035), the Research Council of Norway (project 221899), the University of Victoria, and the Norwegian University of Science and Technology and is gratefully acknowledged. Thomas Holm thanks the Faculty of Natural Sciences and Technology at Norwegian University of Science and Technology for the award of a scholarship. 


\section{Bibliography}

1. D. D. Macdonald, Mod. Asp. Electroc., 11, 141 (1975).

2. G. Wildgoose, D. Giovanelli, N. Lawrence and R. G. Compton, Electroanalysis, 16, 421 (2004).

3. P. Gründler, A. Kirbs and L. Dunsch, ChemPhysChem, 10, 1722 (2009).

4. P. Gründler, In-situ Thermoelectrochemistry, Springer Heidelberg, New York (2015).

5. R. Cowan and A. Kaznoff, Corrosion, 29, 123 (1973).

6. B. Case and G. Bignold, J. Appl. Electrochem., 1, 141 (1971).

7. D. D. Macdonald, Corrosion, 34, 75 (1978).

8. D. D. Macdonald, A. Scott and P. Wentrcek, J. Electrochem. Soc., 126, 908 (1979).

9. S. Hettiarachchi, P. Kedzierawski and D. D. Macdonald, J. Electrochem. Soc., 132, 1866 (1985).

10. D. Midgley, Talanta, 37, 767 (1990).

11. W. Bogaerts and A. van Haute, J. Electrochem. Soc., 131, 68 (1984).

12. A. McDonald, F.-R. Fan and A. J. Bard, J. Phys. Chem., 90, 196 (1986).

13. L. Trevani, E. Calvo and H. Corti, Electrochem. Commun., 2, 312 (2000).

14. Y. Chen, M. Urquidi-Macdonald and D. D. Macdonald, J. Nucl. Mater., 348, 133 (2006).

15. A. J. Bard, W. Flarsheim and K. Johnston, J. Electrochem. Soc., 135, 1939 (1988).

16. H. Nonaka and Y. Matsumura, J. Electroanal. Chem., 520, 101 (2002).

17. A. P. M. Carnargo, B. A. F. Previdello, H. Varela and E. R. Gonzales, Quim. Nova, 33, 2143 (2010).

18. P. K. Dahlstrøm, Electro-oxidation of small organic molecules, Ph.D. thesis, Norwegian University of Science and Technology (2012).

19. M. Umeda, H. Sugii and I. Uchida, J. Power Sources, 179, 489 (2008).

20. D. Bokach, J. L. G. de la Fuente, M. Tsypkin, P. Ochal, I. C. Endsjø, R. Tunold, S. Sunde and F. Seland, Fuel Cells, 11, 735 (2011). 